\title{
Asphericity of galaxy clusters and Sunyaev-Zel'dovich effect
}

\author{
D. Puy ${ }^{1,2}$, L. Grenacher ${ }^{1,2}$, P. Jetzer ${ }^{1,2}$, and M. Signore ${ }^{3}$ \\ 1 Paul Scherrer Institute, LAP, 5232 Villigen PSI, Switzerland \\ 2 Institute of Theoretical Physics, University of Zürich, Switzerland \\ ${ }^{3}$ Observatoire de Paris, DEMIRM, Paris, France
}

A\&A, 363, 415-424 (2000)

Equations (B.3), (B.4) and (B.6) in Appendix B must be replaced by the following expressions:

$I_{\mathrm{SZ}}^{\mathrm{rot}}\left(r_{x}, r_{z}, \theta\right)=$

$\frac{1}{\sqrt{\frac{\cos ^{2} \theta}{\zeta_{2}^{2}}+\frac{\sin ^{2} \theta}{\zeta_{1}^{2}}}}\left(1+\eta_{\theta}\right)^{-\frac{3}{2} \beta+\frac{1}{2}} B\left(\frac{3}{2} \beta-\frac{1}{2}, \frac{1}{2}\right)$,

with

$\eta_{\theta}=\frac{r_{x}^{2}}{\zeta_{1}^{2} \cos ^{2} \theta+\zeta_{2}^{2} \sin ^{2} \theta}+\frac{r_{z}^{2}}{\zeta_{3}^{2}}$

$n_{\mathrm{e}}^{\mathrm{proj}}\left(r_{x}, r_{y}, r_{z}\right)=n_{\mathrm{eo}}\left[1+\frac{r_{x}^{2}}{\tilde{\zeta}_{1}^{2}}+\frac{r_{y}^{2}}{\tilde{\zeta}_{2}^{2}}+\frac{r_{z}^{2}}{\tilde{\zeta}_{3}^{2}}\right]^{-3 \beta / 2}$,

where $\tilde{\zeta}_{1}$ is the maximum value that we get along the $r_{x}$ axis in units of $r_{\mathrm{c}}$

$\tilde{\zeta}_{1}=\sqrt{\zeta_{1}^{2} \cos ^{2} \theta+\zeta_{2}^{2} \sin ^{2} \theta}$,

moreover, $\tilde{\zeta}_{3} / \tilde{\zeta}_{2}=\zeta_{3} / \zeta_{2}=1$ (prolate).

$I_{\mathrm{SX}}^{\mathrm{rot}}\left(r_{x}, r_{z}, \theta\right)=$

$\frac{1}{\sqrt{\frac{\cos ^{2} \theta}{\zeta_{2}^{2}}+\frac{\sin ^{2} \theta}{\zeta_{1}^{2}}}}\left(1+\eta_{\theta}\right)^{-3 \beta+\frac{1}{2}} B\left(3 \beta-\frac{1}{2}, \frac{1}{2}\right)$.

Thus also Figs. 7 and 8 get modified:

In Figs. 7 and 8 we have plotted the relative error due to the projection effects as a function of the rotation angle $\theta$ and the axes ratio $\zeta_{1} / \zeta_{3}$ of the ellipsoid. It is a pure geometrical effect and thus the same for the $y$ parameter, the surface brightness and the Hubble constant. The maximal $\theta$ is assumed to be $\pi / 4 \sim 0.785$, for which we find an underestimation of almost $17 \%$. The influence of the axes ratio turns out to be less than $5 \%$.

Acknowledgements. We thank Rocco Piffaretti for fruitful comments on the original version of this paper.

Send offprint requests to: D. Puy, e-mail: puy@physik.unizh.ch

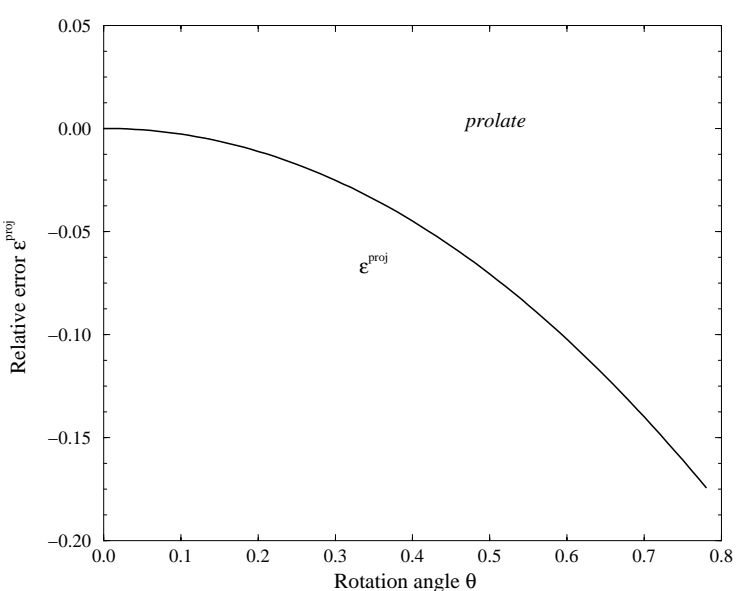

Fig. 7. The relative error, when comparing a projected and a rotated prolate-shaped cluster, is shown as a function of $\theta$. The axes ratio is fixed to be $\zeta_{1} / \zeta_{3}=1.5$

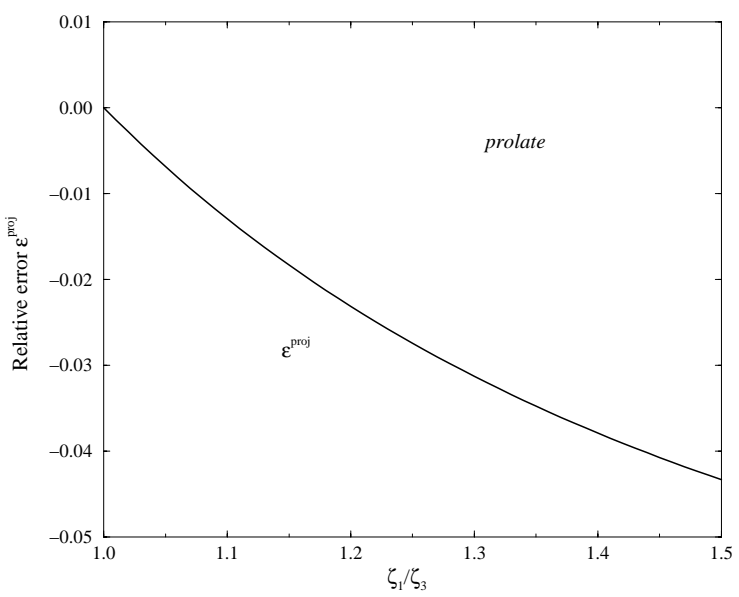

Fig. 8. The relative error between a projected and a rotated prolate-shaped cluster profile is shown as a function of the axes ratio $\zeta_{1} / \zeta_{3}$. The rotation angle $\theta$ is fixed at $\pi / 4$ 